\title{
Prescribing in renal supportive care
}

\section{SUMMARY}

Renal supportive care incorporates the principles of palliative care into the management of patients with advanced kidney disease. Its focus is on improving the quality of life for patients with a high burden of symptoms.

Common problems include pain, restless legs syndrome and uraemic pruritus. Symptom management must involve patient participation, education and non-pharmacological strategies to address both physical and psychosocial problems, and to prioritise patient-centred goals.

The patients are medically complex and polypharmacy is common. When prescribing, it is important to consider the altered pharmacokinetics, potential drug interactions and the clearance of drugs by dialysis.

\section{Introduction}

Renal supportive care is a relatively new activity that incorporates the principles of specialist palliative care within the standard care of patients with advanced chronic kidney disease. This is relevant for patients receiving haemodialysis or peritoneal dialysis who have a high burden of physical and psychological symptoms. It is also suitable for patients with endstage kidney disease who are being conservatively managed without dialysis.

Patients needing renal supportive care tend to be older, have a high symptom burden and multiple comorbidities. Patient-centred goals, such as enhancing quality of life, symptom management and psychosocial support, are therefore the priorities of care. Treatment strategies must be flexible, practical and holistic, incorporating non-pharmacological and pharmacological options and addressing multiple facets including physical, psychosocial and spiritual domains.

\section{General prescribing principles}

Prescribing drugs in renal supportive care can be challenging. End-stage kidney disease alters the pharmacokinetics of renally eliminated drugs, leading to a risk of accumulation and toxicity. Adjusting doses and dosing intervals is necessary to ensure safety while maintaining efficacy. Some commonly used drugs such as non-steroidal anti-inflammatory drugs (NSAIDs) are contraindicated in end-stage kidney disease. Multiple comorbidities lead to polypharmacy, and drug interactions are common. Prescribing differs for haemodialysis, peritoneal dialysis and conservative management because some drugs can be removed by haemodialysis or (less commonly) peritoneal dialysis.

Most drugs with significant renal elimination must be used cautiously but are not always contraindicated.
A general rule is to start with the lowest dose, use longer dosing intervals and increase the dose slowly while monitoring for efficacy and features of toxicity. Drugs cleared by haemodialysis should be given after haemodialysis.

\section{Common symptoms}

Symptoms place a large burden on patients with advanced kidney disease and their families. Treatments should be directed towards the patient's priorities, take account of their preferences and be feasible. The goals should be achievable.

\section{Pain}

Pain is common in chronic kidney disease and usually attributable to one or more comorbidities. It is helpful to distinguish nociceptive pain caused by tissue injury from neuropathic pain caused by nerve damage, giving a tingling, burning, stabbing or shooting sensation. The experience and impact of pain varies between patients. Chronic pain is often associated with significant physical and psychosocial consequences.

Treatment strategies must incorporate education, patient participation and evaluation. They should focus on patient-centred goals, especially if the underlying pathology cannot be corrected. If possible, the cause of the pain should be identified, as some causes have specific therapy, such as urate lowering for gout, facet joint injections, or antiangina drugs for coronary ischaemia.

\section{Non-drug therapy}

For localised pain, heat and cold packs are helpful, as are joint splints or a walking aid. Physiotherapy, hydrotherapy, exercises (both gentle aerobic and resistance training) ${ }^{1}$ and weight reduction are effective for chronic musculoskeletal pain.
Kelly Li

Staff specialist nephrologist

\section{Mark Brown}

Senior staff nephrologist and Obstetric medicine physician

Medical director

Professor ${ }^{2}$

'Renal Medicine, St George Hospital

${ }^{2}$ Renal Medicine, University of NSW

Sydney

\section{Keywords}

end-stage kidney disease, palliative care

Aust Prescr 2020;43:57-60 https://doi.org/10.18773/ austprescr.2020.004 


\section{Drug therapy}

Systemic NSAIDs are contraindicated, but a topical NSAID such as diclofenac can be used for localised musculoskeletal pain.

Systemic treatment should follow the World Health Organization analgesic ladder, ${ }^{2}$ with a stepwise approach beginning with non-opioids, and progressing to opioids with adjuvants. Paracetamol is the initial analgesic of choice in chronic kidney disease. There is no dose modification and paracetamol remains a useful background treatment even when opioids are required. Opioids must be used carefully in renal supportive care, given their narrow therapeutic window and potential for accumulation and toxicity (Table). ${ }^{3,4}$ For moderate to severe pain that has not responded to non-opioid drugs and is detrimental to physical function and quality of life, short-acting opioids can be considered. They are started at a low dose and slowly titrated up according to pain relief and adverse effects. ${ }^{3}$

Adjuvant therapy should be added for severe or refractory nociceptive pain, or used as initial therapy for neuropathic pain. Gabapentin and pregabalin, calcium channel alpha-2-delta ligands (or gabapentinoids), are efficacious and have multiple uses in renal supportive care. They are the preferred initial therapy for neuropathic pain. Due to their almost exclusive renal elimination, substantial dose reductions are needed. Monitoring for the common adverse effects of somnolence, dizziness and gait disturbance is important. Start therapy with gabapentin $100 \mathrm{mg}$ or pregabalin $25 \mathrm{mg}$ on alternate nights for conservative management and peritoneal dialysis, and three times weekly after haemodialysis for patients having haemodialysis. ${ }^{3}$ Increases to the dosing frequency (to nightly and twice daily) or the dose (up to gabapentin $300 \mathrm{mg}$ or pregabalin $75 \mathrm{mg} / 24$ hours) should occur one week apart while monitoring for adverse effects. Higher doses may be tolerated in some patients, but specialist advice should be sought.

Tricyclic antidepressants, such as amitriptyline, can be used to manage neuropathic pain. ${ }^{3}$ Serotonin and norepinephrine reuptake inhibitors such as duloxetine can also be used. ${ }^{3,5}$

\section{Table Opioid use in end-stage kidney disease}

\begin{tabular}{|c|c|c|c|c|}
\hline Opioid & Renal clearance & Formulation & Starting dose & Comments \\
\hline Hydromorphone & $\begin{array}{l}\text { Small amount } \\
\text { excreted in urine: } \\
\text { needs dose reduction } \\
\text { Cleared by } \\
\text { haemodialysis }\end{array}$ & $\begin{array}{l}\text { Oral: } \\
\text { - liquid } \\
\text { - immediate-release tablet } \\
\text { - sustained-release tablet }\end{array}$ & $\begin{array}{l}\text { Immediate-release oral } \\
\text { hydromorphone starting at } \\
0.5 \text { mg as required } 4 \text { times } \\
\text { daily as liquid formulation } \\
\text { Dose after haemodialysis }\end{array}$ & $\begin{array}{l}\text { Used for severe acute pain. Long-acting } \\
\text { oral hydromorphone can accumulate } \\
\text { and lead to toxicity and should be } \\
\text { used only when a stable daily dose is } \\
\text { established (or switch to fentanyl patch } \\
\text { instead for maintenance). }\end{array}$ \\
\hline Oxycodone & $\begin{array}{l}\text { Small amount } \\
\text { excreted in urine: } \\
\text { needs dose reduction } \\
\text { Cleared by } \\
\text { haemodialysis }\end{array}$ & $\begin{array}{l}\text { Oral: } \\
\text { - immediate-release tablet } \\
\text { - sustained-release, with } \\
\text { or without naloxone }\end{array}$ & $\begin{array}{l}\text { Immediate-release oral } \\
\text { oxycodone starting at } \\
2.5 \text { mg as required } 4 \text { times } \\
\text { daily } \\
\text { Dose after haemodialysis }\end{array}$ & $\begin{array}{l}\text { Used for severe acute pain. Start slow } \\
\text { release only after a stable daily dose is } \\
\text { established. }\end{array}$ \\
\hline Fentanyl & $\begin{array}{l}\text { Minimal renal } \\
\text { elimination: no dose } \\
\text { reduction required }\end{array}$ & Patch applied to skin & $\begin{array}{l}\text { Not recommended in } \\
\text { opioid-naïve patients }\end{array}$ & $\begin{array}{l}\text { Onset of action usually 8-12 hours after } \\
\text { first patch application. } \\
\text { Useful for background analgesia once } \\
\text { pain controlled with hydromorphone or } \\
\text { oxycodone. }\end{array}$ \\
\hline Buprenorphine & $\begin{array}{l}\text { Minimal renal } \\
\text { elimination: no dose } \\
\text { reduction required }\end{array}$ & Patch applied to skin & $\begin{array}{l}\text { Start with } 5 \text { mg patch } \\
\text { changed every week }\end{array}$ & $\begin{array}{l}\text { Onset of action usually } 12-24 \text { hours } \\
\text { after initial patch application. Useful for } \\
\text { background analgesia. Can be started } \\
\text { in opioid-naïve patients. }\end{array}$ \\
\hline
\end{tabular}

\section{Additional notes:}

Morphine and codeine are best avoided in renal supportive care as active metabolites accumulate in renal failure and lead to clinically significant toxicity including sedation, confusion, myoclonus, and respiratory depression.

Tramadol (maximum 50 mg twice a day) and tapentadol (maximum 50 mg twice a day) can be used at low doses and with caution due to multiple potential drug interactions, unpredictable risk of overdose, and the risk of serotonin syndrome with concomitant use of some antidepressants. May provide additional benefits in neuropathic pain. ${ }^{4}$

Methadone has minimal renal elimination and can be used in renal supportive care without dose adjustment. However, due to its long half-life, specialist advice is recommended when starting methadone in the community. ${ }^{4}$

Source: references 3 and 4 


\section{Restless legs syndrome}

Restless legs syndrome is a sensorimotor disorder characterised by an overwhelming urge to move the legs, predominantly during periods of inactivity. It is temporarily relieved by movement. Patients typically describe achy, creeping, crawling or itchy sensations in the legs. ${ }^{6}$ Restless legs syndrome is prevalent in patients having dialysis (12-25\%) but also affects other patients with chronic kidney disease. It is associated with reduced quality of life, anxiety, insomnia, daytime sleepiness and premature stopping of dialysis.?

\section{Non-drug therapy}

Aerobic exercises such as walking and stretching may be helpful. Exacerbating substances such as nicotine, alcohol, and caffeine should be avoided.

\section{Drug therapy}

Avoid drugs such as dopamine antagonists (typically antipsychotics and metoclopramide), antihistamines and serotonergic antidepressants. Correcting iron deficiency may be helpful.

Gabapentinoids are first-line drug therapy for restless legs syndrome. An extra dose can be taken one hour before haemodialysis if the patient is symptomatic during haemodialysis.

Non-ergot dopamine agonists are also efficacious for restless legs syndrome.? Ropinirole (compared to pramipexole) has less accumulation in renal failure and can be started at $0.25 \mathrm{mg}$ at night and titrated up to $2 \mathrm{mg}$ at night.

\section{Uraemic pruritus}

Uraemic pruritus is an itch affecting large bilateral symmetrical surface areas with no associated primary skin lesion. It can be generalised or localised to the back, face and arms. ${ }^{8}$ Uraemic pruritus is associated with depression and reduced quality of life, and exacerbates sleep problems. ${ }^{6}$ Non-uraemic causes of pruritus, such as dry skin, drug reactions, scabies or fungal skin infections, should not be overlooked.

Good skin care is essential, ${ }^{6}$ as dry skin exacerbates itch. It is helpful to avoid long, hot showers and harsh soaps, and to moisturise within minutes of washing while the skin is still damp. Aqueous cream emollient and baby oil are effective in reducing uraemic pruritus and improving quality of life if applied 2-4 times daily. ${ }^{6}$ If the itch is localised, capsaicin $0.025 \%$ can be applied topically. Although effective, it can cause burning $^{8}$ and applying topical menthol beforehand may improve tolerability.

In more generalised uraemic pruritus, gabapentinoids have the strongest supporting evidence..$^{8,9}$ Alternatives include sertraline 50 mg daily, doxepin
$10 \mathrm{mg}$ twice daily and evening primrose oil (affects gamma linoleic acid) starting at one capsule (1000 mg) at night, up to two capsules twice daily. ${ }^{6}$

For unresponsive uraemic pruritus, non-uraemic causes need to be reconsidered. Once these are excluded, treatment with ultraviolet B light can be effective. ${ }^{8}$

\section{Fatigue}

In renal supportive care fatigue is the most common symptom. Its cause is multifactorial, so management involves identifying and addressing contributing factors:

- iron deficiency or the anaemia of chronic kidney disease - can be corrected with iron supplements and erythropoietin-stimulating drugs

- vitamin D deficiency - can be managed with oral supplementation

- metabolic acidosis - should be corrected with oral sodium bicarbonate

- mood disorders such as anxiety and depression should be assessed and treated

- obstructive sleep apnoea - should be assessed and treated

- $\quad$ sleep disturbances

- drugs that exacerbate fatigue, including benzodiazepines, gabapentinoids, beta blockers, centrally acting antihypertensives and sedating antidepressants (such as mirtazapine).

After addressing reversible factors, patients should be counselled regarding maintaining good nutrition, regular exercise and practical energy conservation strategies. For patients taking drugs causing fatigue, management needs to be negotiated with the patients as to the indications for these drugs, alternatives and treatment goals.

\section{Sleep disturbances}

A variety of symptoms can contribute to poor sleep. These include restless legs syndrome, uraemic pruritus, anxiety, depression, nocturia and chronic pain. These should be explored and treated when possible. Nocturia can be managed by taking diuretics early in the day and avoiding fluid, alcohol and caffeine in the evenings. In men, treat any comorbid prostate pathology.

Educating patients regarding good sleep hygiene can foster self-management. Sleep hygiene and cognitive behavioural therapy should be the mainstay of treatment. Drugs such as temazepam and zopiclone should be limited to short-term use. Melatonin is another option, but its efficacy is also limited to short-term use. ${ }^{10}$ 


\section{Nausea}

While metabolic disturbances in uraemia can cause nausea, other contributing factors are common:

- drugs, particularly opioids, dopamine agonists and some antidepressants

- gastroparesis, commonly from comorbid diabetes, can lead to delayed gastric emptying, and worsening reflux symptoms

- constipation.

\section{Non-drug therapy}

Smaller, frequent meals, good oral hygiene and maintaining an upright posture after meals to minimise reflux are important. Constipation should be managed.

\section{Drug therapy}

Dopamine antagonists, such as domperidone, have prokinetic effects and are best given 30 minutes before meals. Metoclopramide or low-dose haloperidol (0.5-1 mg) can be substituted and have additional CNS effects on nausea. However, they can have extrapyramidal adverse effects during longterm use and should be avoided in patients with restless legs syndrome, especially if they are taking dopamine agonists. Antihistamines such as cyclizine, and serotonin $\left(5 \mathrm{HT}_{3}\right)$ antagonists such as ondansetron may be used but can be costly for patients.

\section{Taste changes}

Common changes in chronic kidney disease include a metallic or bitter taste, lack of taste in food, and a dry mouth. This can affect appetite, nutrition and the enjoyment of food.
Sodium bicarbonate mouthwash can improve taste and dry mouth. It is cheap and simple to make - one teaspoon of sodium bicarbonate in $500 \mathrm{~mL}$ water. This mouthwash should be used regularly during the day, usually every four hours. Other helpful habits include:"11

- a glass of soda water before meals

- avoiding foods that give bitter tastes such as red meat, and tea or coffee

- adding sweet or sour flavours such as sugar, vinegar, fruits, or lemon to relieve bitterness

- $\quad$ adding herbs and spices, including chilli, to give extra flavour to food

- peppermints and chewing gums to help stimulate saliva and improve taste.

\section{Conclusion}

Prescribing for patients receiving renal supportive care requires a flexible and nuanced approach, taking into consideration altered pharmacokinetics, polypharmacy, comorbidities and practicality. Management of these patients' complex care requires using both non-pharmacological and pharmacological therapies focussing on patientcentred goals. $<$

Conflict of interest: none declared

\section{REFERENCES}

1. Wewege M, Booth J, Parmenter B. Aerobic vs. resistance exercise for chronic non-specific low back pain: a systematic review and meta-analysis. J Back Musculoskelet Rehabil 2018;31:889-99. https://doi.org/10.3233/BMR-170920

2. WHO Expert Committee on Cancer Pain Relief and Active Supportive Care \& World Health Organization. Cancer pain relief and palliative care: report of a WHO expert committee [meeting held in Geneva from 3 to 10 July 1989]. WHO Technical Report Series 804. Geneva: World Health Organization; 1990. https://apps.who.int/iris/ handle/10665/39524

3. Davison SN, Koncicki H, Brennan F. Pain in chronic kidney disease: a scoping review. Semin Dial 2014;27:188-204. https://doi.org/10.1111/sdi.12196

4. Davison SN, Tupala B, Wasylynuk BA, Siu V, Sinnarajah A, Triscott J. Recommendations for the care of patients receiving conservative kidney management: focus on management of chronic kidney disease and symptoms. Clin J Am Soc Nephrol 2019:14:626-34. https://doi.org/ 10.2215/CJN.10510917

5. Lunn MP, Hughes RA, Wiffen PJ. Duloxetine for treating painful neuropathy, chronic pain or fibromyalgia. Cochrane Database Syst Rev 2014:CD007115. https://doi.org/ 10.1002/14651858.CD007115.pub3
6. Scherer JS, Combs SA, Brennan F. Sleep disorders, restless legs syndrome, and uremic pruritus: diagnosis and treatment of common symptoms in dialysis patients. Am J Kidney Dis 2017:69:117-28. https://doi.org/10.1053/j.ajkd.2016.07.031

7. Novak M, Winkelman JW, Unruh M. Restless legs syndrome in patients with chronic kidney disease. Semin Nephrol 2015;35:347-58. https://doi.org/10.1016/ j.semnephrol.2015.06.006

8. Simonsen E, Komenda P, Lerner B, Askin N, Bohm C, Shaw J, et al. Treatment of uremic pruritus: a systematic review. Am J Kidney Dis 2017;70:638-55. https://doi.org/10.1053/ j.ajkd.2017.05.018

9. Lau T, Leung S, Lau W. Gabapentin for uremic pruritus in hemodialysis patients: a qualitative systematic review. Can J Kidney Health Dis 2016;3:14. https://doi.org/10.1186/ s40697-016-0107-8

10. Russcher M, Koch BC, Nagtegaal JE, van Ittersum FJ, Pasker-de Jong PC, Hagen EC, et al. Long-term effects of melatonin on quality of life and sleep in haemodialysis patients (Melody study): a randomized controlled trial. Br J Clin Pharmacol 2013;76:668-79. https://doi.org/10.1111/ bcp.12093

11. NSW Renal Dieticians. Managing taste changes in advanced CKD. Renal \& Hypertension Service, St George \& Sutherland Hospitals; 2017. https://stgrenal.org.au/renal-supportivecare\#Nutrition [cited 2020 Mar 1] 